\title{
Modelling an influenza pandemic: A guide for the perplexed
}

\author{
Pandemic Influenza Outbreak Research Modelling Team (Pan-InfORM)
}

$\infty \quad$ See other H1N1 articles: Editorial, page 123; Research, page 159

A new swine-origin influenza A (H1N1) virus, initially identified in Mexico, has now caused outbreaks of disease in at least 74 countries, with declaration of a global influenza pandemic by the World Health Organization on June 11, 2009. ${ }^{1}$ Optimizing public health responses to this new pathogen requires difficult decisions over short timelines. Complicating matters is the unpredictability of influenza pandemics: planners cannot base their decisions solely on pre-pandemic factors or on experience from earlier pandemics. We suggest that mathematical modelling can inform and optimize health policy decisions in this situation.

\section{Uses of models}

Mathematical models of infectious diseases are useful tools for synthesizing the best available data on a new pathogen, comparing control strategies and identifying important areas of uncertainty that may be prioritized for urgent research.

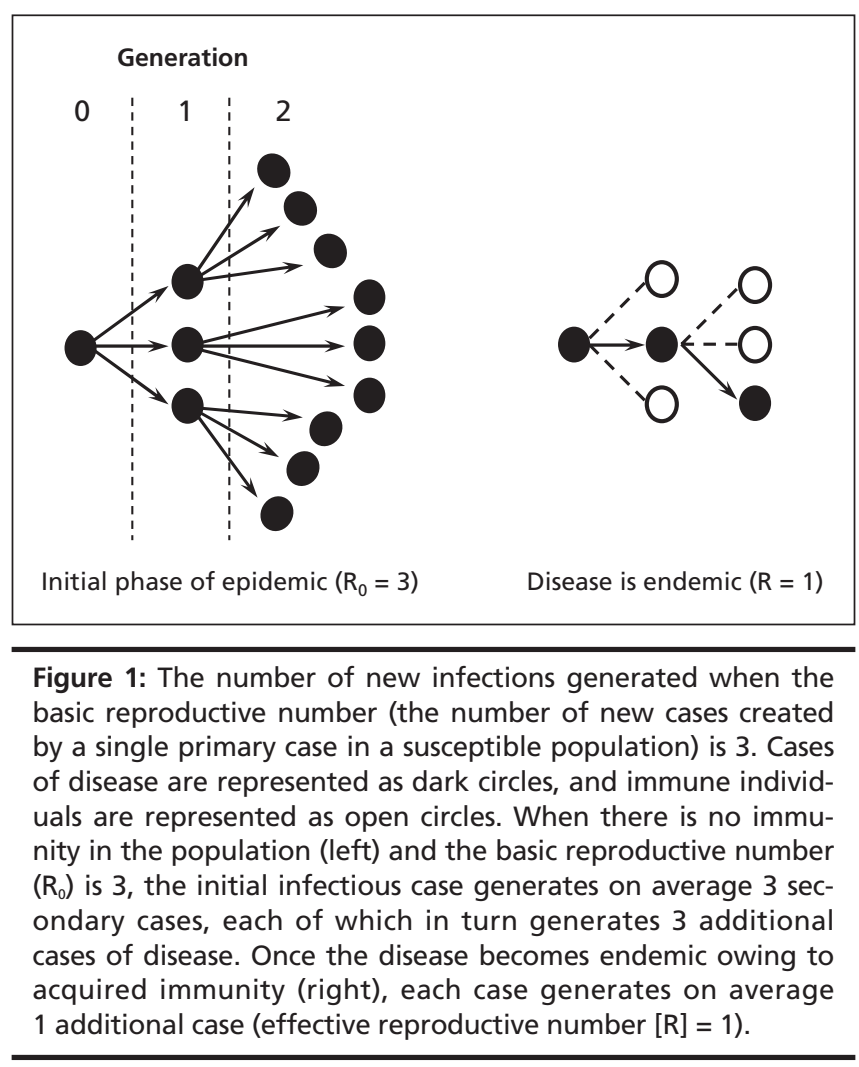

\section{Key points}

- When a new infectious disease emerges, mathematical models can project plausible scenarios, guide control strategies and identify important areas for urgent research.

- Models of influenza pandemics suggest roles for antiviral drugs and vaccines. Models also raise concerns about antiviral resistance.

- Knowledge translation is a key part of modelling activities that aim to optimize policy decisions for containment of new infectious diseases.

As an example of synthesizing data, consider the process of developing a mathematical model of the effectiveness of influenza vaccines: modellers must draw together information on influenza epidemiology (including patterns of spread in different age groups), the natural history of influenza, the effectiveness of vaccines in randomized trials and the duration of immunity following vaccination or natural infection, ${ }^{2,3}$ which cannot all be derived from a single study. Once the model is developed, rapid and inexpensive "experiments" can be performed by simulating alternative vaccination strategies (e.g., vaccinating children most likely to transmit influenza, or vaccinating older adults most likely to have severe complications of influenza). ${ }^{2}$

The uncertainty involved in this process can be assessed through sensitivity analysis (in this case, by varying estimates of vaccine effectiveness across plausible ranges) to examine whether such variation results in markedly different outcomes. Uncertain model inputs that are extremely influential in determining the best course of action should be prioritized for future research.

\section{Elements of models}

Elements of epidemic models often include "compartments" or "states" that describe the susceptibility, infectiousness or immunity of individuals in a population, and "parameters" (numbers) that describe how individuals move between these states.

A key model parameter is the basic reproductive number, referred to as $\mathrm{R}_{0}{ }^{4}$ This is the number of new, secondary infections created by a single primary infectious case introduced into a totally susceptible population (Figure 1). The importance of $R_{0}$ relates to the information it provides to planners: $\mathrm{R}_{0}$ determines the potential of a new pathogen to 
cause an epidemic (if $\mathrm{R}_{0}$ is greater than 1). It can also be used to estimate the final size of the epidemic with and without control measures (Figure 2). Models provide a systematic way to estimate $\mathrm{R}_{0}$, which reflects the product of duration of infectiousness, contact numbers per unit time and infectiousness per contact. As each of these quantities increases, $\mathrm{R}_{0}$ increases.

$\mathrm{R}_{0}$ is "unit-less" with respect to time. Therefore, knowing how fast a disease spreads requires knowledge of "generation times." A generation is the average time from the onset of infectiousness in a case to the onset of infectiousness in a person that the case has infected. Generations can be estimated as the sum of the disease's latent (infected, but noninfectious) periods and half the duration of infectiousness. Measles and tuberculosis both have similar (very high) $R_{0}$ values. However, measles is more infectious per contact, and tuberculosis has a much longer generation time because of a longer duration of infectiousness and a prolonged latent period.

Diseases that have low $\mathrm{R}_{0}$ values but short generation times can have relatively rapid increases in the number of cases. Published estimates suggest that the $\mathrm{R}_{0}$ for the new influenza A (H1N1) virus is 1.5 (slightly lower than estimates for the 1918 influenza pandemic $\left.{ }^{5}\right){ }^{6}$ The generation time is estimated to be about 2 days, ${ }^{6}$ although preliminary unpublished estimates in the province of Ontario suggest longer generation times (Rachel Savage, Ontario Agency for Health Protection and Promotion, personal communication, May 2009).

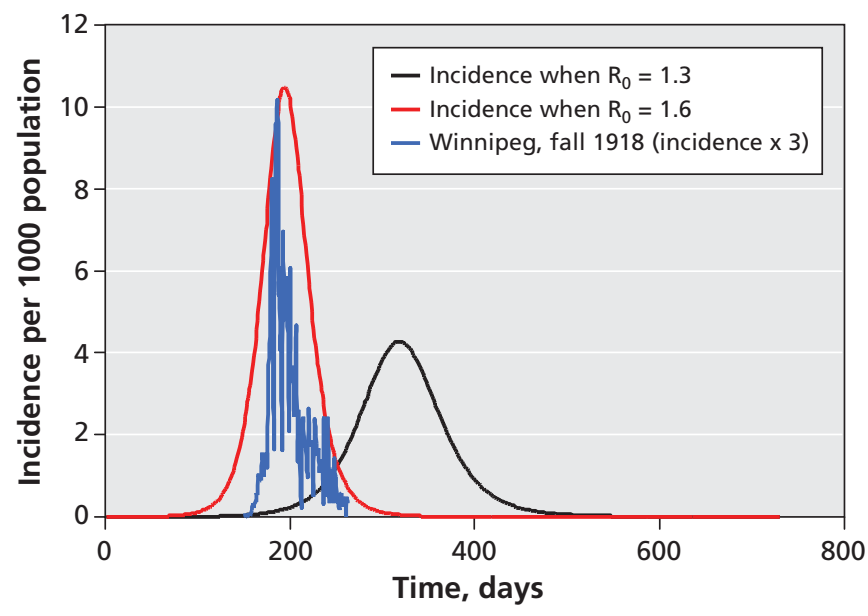

Figure 2: The effect of changing the basic reproductive number $\left(R_{0}\right)$ on the severity and duration of an influenza epidemic. A higher $R_{0}$ (1.6, red curve) results in an epidemic with a higher peak incidence and a greater cumulative attack rate (not shown). When the $R_{0}$ is lower (1.3, black curve), the epidemic peaks later, lasts longer and has a lower cumulative attack rate. The basic reproductive number of the disease is reduced through control interventions, including isolation of cases and "social distancing" (e.g., school closures). The blue curve represents actual incidence data from the autumn wave of the 1918 influenza pandemic in Winnipeg (multiplied by 3 for comparability of scales).

\section{The new influenza strain and seasonality}

Mathematical models have provided important insights into the seasonality of influenza by showing that small seasonal variations in viral transmissibility can drive large annual surges in the incidence of the disease. ${ }^{3}$ The 1918 influenza pandemic began in the spring, was relatively quiescent over the summer months and became more severe the following fall. Models suggest that the new influenza strain could follow a similar pattern in countries in the northern hemisphere (Figure 3). Such models raise concern regarding the potential for the new strain to cause serious epidemics in the near term in the southern hemisphere: South American countries, Australia and New Zealand will enter their upcoming influenza season without a vaccine against this strain.
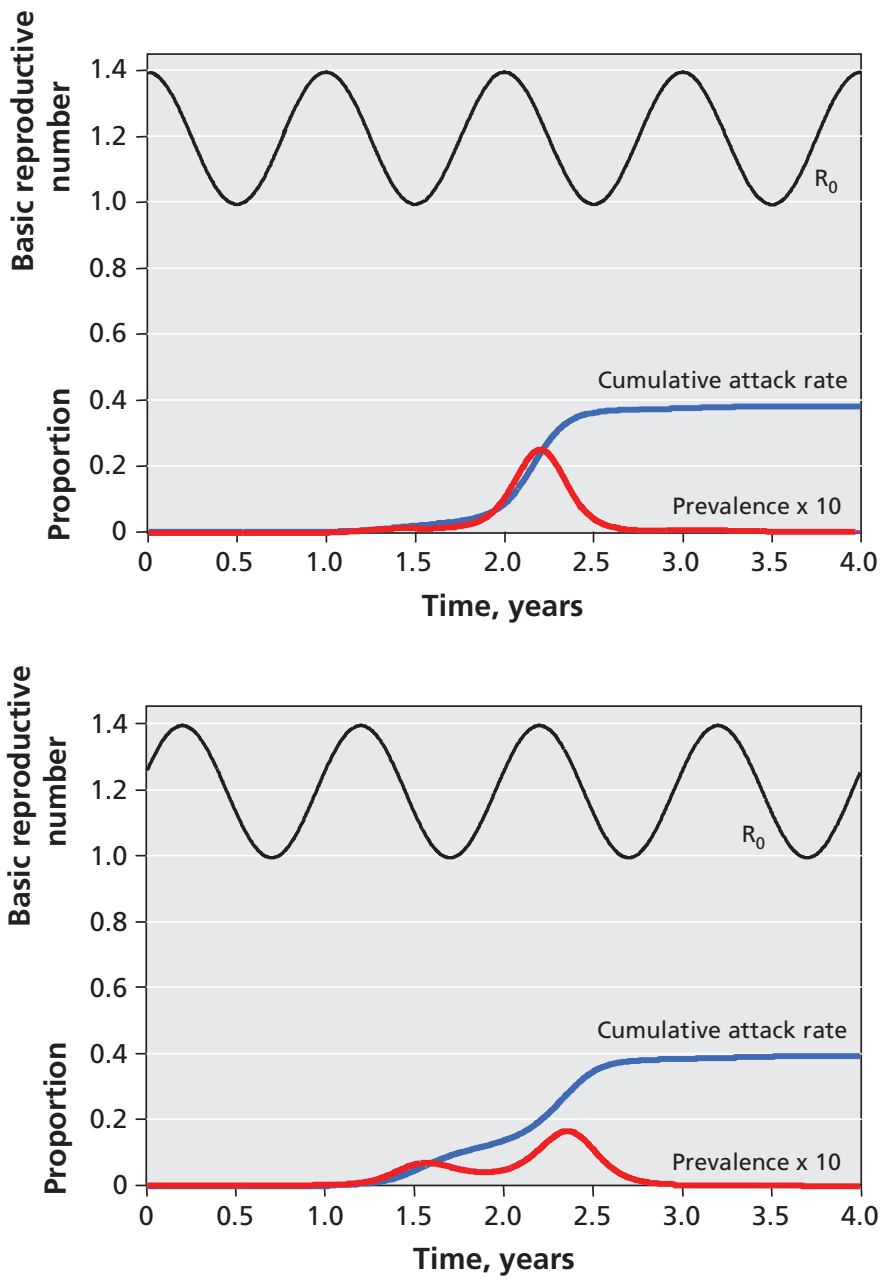

Figure 3: Possible effects of seasonality on a novel influenza strain. Panels show simulated epidemic curves when the basic reproductive number $\left(R_{0}\right)$ for influenza oscillates from 1.4 in winter to just under 1 in summer (black curves). When an epidemic emerges in winter (top panel), it is associated with a monophasic increase in prevalence (red curve). However, springtime emergence (bottom panel) results in a biphasic epidemic (red curve), as happened in the 1918 influenza pandemic. Cumulative attack rates are represented by blue curves. Prevalence data are multiplied by 10 for comparability of scales. 


\section{Mitigation without vaccination}

Nonpharmaceutical interventions considered in published models of influenza transmission include isolation of infectious cases, quarantine of potentially infectious but asymptomatic contacts, and "social distancing" measures such as school closures. ${ }^{7}$ Models suggest that nonpharmaceutical interventions that reduce $\mathrm{R}_{0}$ through different, complementary mechanisms can sometimes be synergistic.

Without a vaccine, antiviral drugs represent an important tool for controlling influenza pandemics. The new influenza strain appears to be susceptible to the neuraminidase inhibitors oseltamivir and zanamivir. Models in which resistance is not considered suggest that the early use of antiviral drugs could contain influenza epidemics caused by strains with low $\mathrm{R}_{0}$ values. When resistance is considered in models, it becomes apparent that limiting the initial use of antiviral drugs, to avoid epidemics caused by antiviral-resistant influenza strains, is the preferred strategy. ${ }^{8}$

\section{Modelling and policy}

Despite the extensive use of models to evaluate epidemics, the integration of modelling into policies for disease control has been limited. ${ }^{9}$ Participants at a 2008 Canadian pandemic planning workshop noted that models are most useful when they are developed cooperatively by modellers and policymakers. The pandemic threat has shown that the ties between modellers and public health decision-makers are closer than originally believed: Canadian modellers, including our group, have helped characterize the epidemiology of this new influenza strain and have provided input to public health authorities on control strategies, including the use of antiviral drugs and school closures.

We hope that models will become more widely available as desktop decision-support tools for front-line public health and medical personnel. Knowledge translation is a key part of modelling activities. Making these models better understood and more accessible will provide a valuable additional weapon in the fight against emerging infectious diseases.

\section{Competing interests: None declared.}

Contributors: All of the authors were involved in the drafting and revision of this manuscript and approved the final version submitted for publication.
Funding: Pan-InfORM is supported by the Canadian Institutes for Health Research, the Natural Sciences and Engineering Research Council of Canada and the Mathematics of Information Technology and Complex Systems (MITACS) network.

\section{REFERENCES}

1. World now at the start of 2009 influenza pandemic [press release]. Geneva (Switzerland): World Health Organization; 2009 June 11. Available: www.who.int/mediacentre /news/statements/2009/h1n1_pandemic_phase6_20090611/en/index.html (accessed 2009 June 22)

2. Dushoff J, Plotkin JB, Viboud C, et al. Vaccinating to protect a vulnerable subpopulation. PLoS Med 2007;4:e174.

3. Dushoff J, Plotkin JB, Levin SA, et al. Dynamical resonance can account for seasonality of influenza epidemics. Proc Natl Acad Sci U S A 2004;101:16915-6.

4. Brauer F. Compartmental models in epidemiology. In: Brauer F, van den Driessche P, Wu J, editors. Mathematical epidemiology. Berlin (Germany): SpringerVerlag; 2008. p. 19-79.

5. Fraser C, Donnelly CA, Cauchemez S, et al. Pandemic potential of a strain of Influenza A (H1N1): early findings. Science 2009 May 14. [Epub ahead of print]

6. Mills CE, Robins JM, Lipsitch M. Transmissibility of 1918 pandemic influenza Nature 2004;432:904-6.

7. Halloran ME, Ferguson NM, Eubank S, et al. Modeling targeted layered containment of an influenza pandemic in the United States. Proc Natl Acad Sci U S A 2008;105:4639-44

8. Moghadas SM, Bowman CS, Röst G, et al. Population-wide emergence of antiviral resistance during pandemic influenza. PLoS ONE 2008;3:e1839.

9. Moghadas S, Pizzi N, Wu J, et al. Managing public health crises: the role of models in pandemic preparedness. Influenza Other Respir Viruses 2009;3:75-9.

Correspondence to: Dr. David Fisman, Ontario Agency for Health Protection and Promotion, Epidemiology and Surveillance, 480 University Ave., Rm. 406, Toronto ON M5G 1V2; fax 647 260-7600; david.fisman@oahpp.ca

Members of the Pandemic Influenza Outbreak Research Modelling Team (Pan-InfORM): Seyed Moghadas PhD, Institute for Biodiagnostics, National Research Council Canada, Winnipeg, Man.; Troy Day PhD, Department of Mathematics and Statistics, Queen's University, Kingston, Ont.; Chris Bauch PhD, Department of Mathematics and Statistics, University of Guelph, Guelph, Ont.; S. Michelle Driedger PhD, Department of Community Health Sciences, University of Manitoba, Winnipeg, Man.; Fred Brauer PhD,

Department of Mathematics, University of British Columbia, Vancouver, BC; Amy L. Greer PhD, Research Institute of The Hospital for Sick Children, Toronto, Ont.; Ping Yan PhD, Public Health Agency of Canada, Ottawa, Ont.; Jianhong Wu PhD, MITACS Centre for Disease Modeling, York University Institute for Health Research, Toronto, Ont.; Nick Pizzi PhD, Institute for Biodiagnostics, National Research Council Canada, Winnipeg, Man.; and David N. Fisman MD MPH, Ontario Agency for Health Protection and Promotion, and the Research Institute of The Hospital for Sick Children, Toronto, Ont. 